\title{
Machining accuracy retainability prediction of machine tool based on least square support vector machine
}

\author{
Qiang Cheng ${ }^{1, a}$, Baobao $\mathrm{Qi}^{1, \mathrm{~b}}$, Bingwei Sun ${ }^{1, \mathrm{c}}$ and Guobin Yan ${ }^{1, \mathrm{~d}}$ \\ ${ }^{1}$ Beijing Key Laboratory of Advanced Manufacturing Technology, Beijing University of Technology, \\ Beijing 100124, China

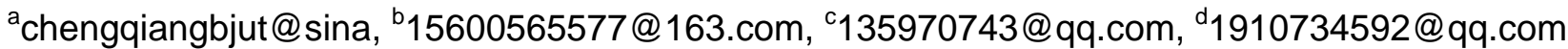

Keywords: machine tool, accuracy retainability, machining accuracy, LS-SVM.

\begin{abstract}
The accuracy retainability is becoming an important performance index of machine tool, and how to improve it is a tough problem faced to manufacturers and users. Generally, it needs to measure the errors termly and repeatedly during the specified period to analyze the timeliness machining accuracy retainability, which generates intricate and vast error data. In this paper, a solution to predict machining accuracy retainability is proposed based on least square support vector machine (LS-SVM). A vertical machining center that machines plane and hole continuously for half a year is selected as an illustrative example. The analysis results show that the proposed method is good at predicting the timeliness machining accuracy retainability of machine tool.
\end{abstract}

\section{Introduction}

As for machine tool, the machining accuracy is an important index to evaluate its performance and characteristics $[1,2]$. In the modern manufacturing industry, the demand for high accuracy and high performance machine tools is also increasing sharply. Therefore, it becomes more and more important to improve the machining accuracy of machine tools[3]. The errors that affect the machining accuracy is mainly including geometric error, thermal error, load error, servo error and interpolation error and so on[4] Among all errors, the geometric errors thermal errors are the main factors affecting the machining precision. According to the characteristics of geometric errors thermal errors, prediction of these errors are an effective way to improve the machining accuracy of machine tools. The geometric error of the machine tool exists in all kinds of mechanical parts, which will eventually be reflected in the machining error of the workpiece. Besides the geometric errors, thermal errors caused by the temperature variations also have a great influence on machining accuracy, especially during continuous and longtime to do the repetitive work or in a temperature-varying workshop. As the machining accuracy is higher and higher, how to keep the machine tool with high accuracy with timeliness is more and more paid attentions there, which is called machining accuracy retention. In general, the machining accuracy retainability of machine tool can be predicted by measurement of comprehensive error in real time. However, by this means, it will generate intricate and vast error data not only because there are many stochastic error sources, but also is very complex and time-consuming. To make the process concise and effective, in this research a machining accuracy retainability prediction method of machine tool is proposed, which can decrease the measurement workload and improve the prediction accuracy.

Therefore, robust and accurate comprehensive error modeling is the first step to predict machine tool's machining accuracy retainability $[5,6]$. In the field of robot research, the screw theory is widely used in recent years. According to robotic geometric property, its motion can be described accurately[7]. In this paper, the screw theory is used to establish the comprehensive error modeling of a 3-axis machine tools.

After the comprehensive error modeling established, how to realize time-varying accuracy retainability prediction is another important step. Support vector machine (SVM) is an effective machine learning method based on statistical learning theory [8]. In this paper, a machine tool' 
machining accuracy retainability prediction method is presented based on Least square support vector machine (LS-SVM).

\section{Timeliness volumetric error modeling}

Error modelling based on POE screw theory. In this research, error modelling is based on POE screw theory [9]. A three-axis machine tool is considered as an example, and its schematic diagram is shown in Figure 1. The topological structure of the machine tool is shown in Figure 2.The errors measured by laser interferometer include many error terms, and the site of error measurement is shown in Figure. 3.

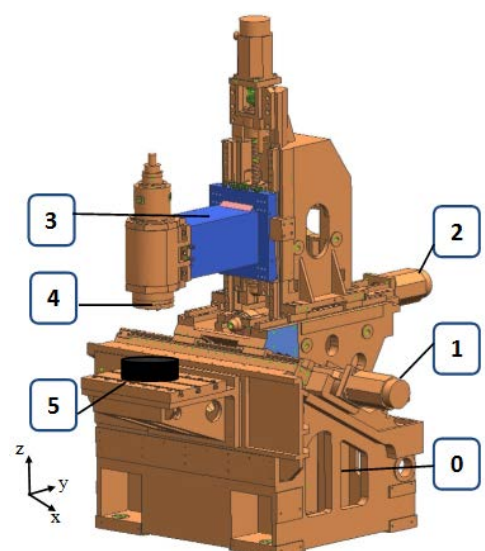

0-bed; 1-Worktable(X-axis); 2—slide carriage(Y-axis);3—RAM (Z-axis);4—cutting tool; 5—work-piece Fig.1 Structure diagram of the 3-axis machining center

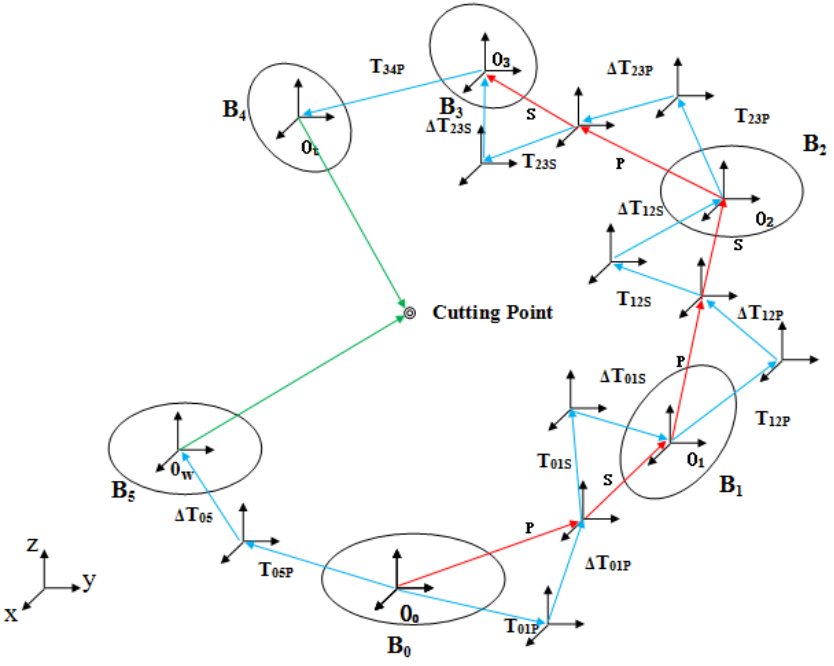

Fig.2 Topological graph of the 3-axis machining center

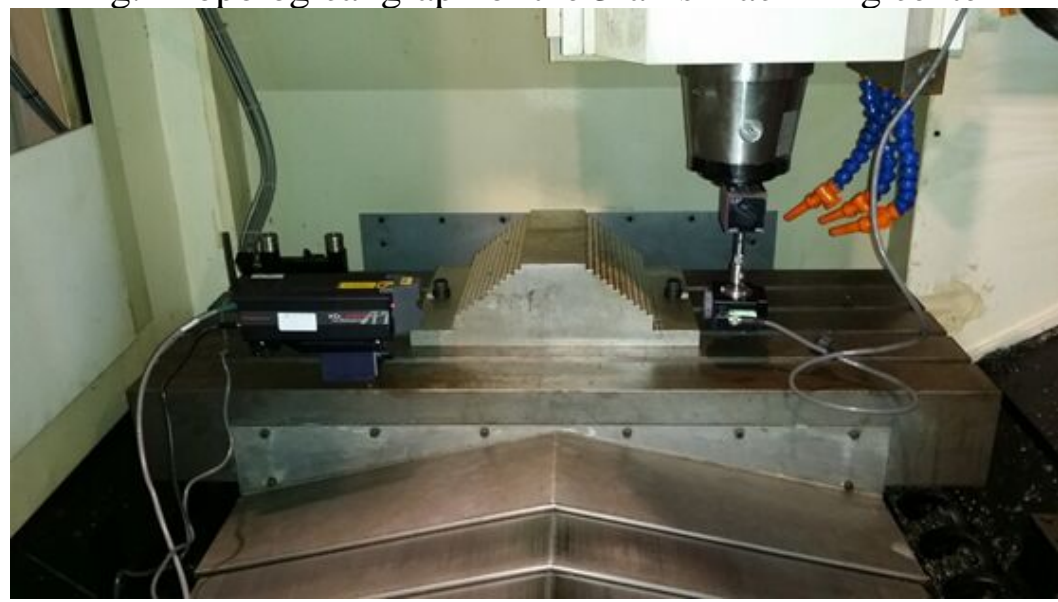

Fig.3 Measurement of errors by laser interferometer

One six-dimensional vector $\Phi$ can also represent the twist, termed the twist coordinates: 
$\Phi=\left[\omega^{\mathrm{T}} v^{\mathrm{T}}\right]^{\mathrm{T}}=\left[\omega_{1}, \omega_{2}, \omega_{3}, v_{1}, v_{2}, v_{3}\right]^{T}$

Assume the vector between the rigid body coordinate system and reference coordinate system is $q$, and the homogeneous transformation matrix of the rigid body is $\boldsymbol{T}=e^{\widehat{\phi} \theta}=\left[\begin{array}{ll}R & \mathrm{q} \\ 0 & 1\end{array}\right]$

The exponential matrix of the twist is also the corresponding homogeneous transformation matrix, namely $\boldsymbol{T}=e^{\widehat{\phi} \theta}$. If $\boldsymbol{\omega}=0$, there is only rigid translational motion, and the homogeneous transformation matrix can be written as:

$$
\boldsymbol{T}=\mathrm{e}^{\widehat{\phi} \theta}=\left[\begin{array}{cc}
I_{3 \times 3} & v \theta \\
0 & 1
\end{array}\right]
$$

If $\omega \neq 0$, which means that the rigid body also has rotational motion, and the homogeneous transformation matrix is:

$$
\boldsymbol{e}^{\widehat{\Phi} \theta}=\left[\begin{array}{cc}
e^{\widehat{\omega} \theta} & \left(I-e^{\widehat{\omega} \theta}\right)\left(\frac{\vec{\omega} \times \vec{v}}{(\|\omega\| \theta)^{2}}\right)+\frac{\vec{\omega}^{T} \vec{v}}{(\|\omega\| \theta)^{2}} \\
0 & 1
\end{array}\right]
$$

The trigonometric series expansion of,$e^{\widehat{\omega} \theta}$ can be expressed as:

$$
e^{\hat{\omega} \theta}=I+\frac{\hat{\omega}}{\|\omega\|} \sin \theta+\frac{\hat{\omega}^{2}}{\|\omega\|^{2}}(1-\cos \theta)
$$

In all of the above, if the screw $\Phi$ is unit, motion of rigid body can be transformed by Eq. (3) and Eq. (4), and Eq. (5) can be described as follows:

$$
\boldsymbol{T}=\boldsymbol{e}^{\widehat{\phi} \theta}=\left\{\begin{array}{cc}
{\left[\begin{array}{cc}
I_{3 \times 3} & \vec{v} \theta \\
O & 1
\end{array}\right]} & \text { if }\|\omega\|=O \\
{\left[\begin{array}{cc}
e^{\hat{\omega} \theta} & \left(I-e^{\hat{\omega} \theta}\right)\left(\frac{\vec{\omega} \times \vec{v}}{(\|\omega\| \theta)^{2}}\right)+\frac{\vec{\omega} \vec{\omega}^{T} \vec{v}}{(\|\omega\| \theta)^{2}} \\
o & 1
\end{array}\right]} & \text { if }\|\omega\|=O
\end{array}\right.
$$

When $\|\omega\| \neq 0$, the rotation angle $\theta=\sqrt{\omega_{1}^{2}+\omega_{2}^{2}+\omega_{3}^{2}}$. Otherwise, the translational distance $\boldsymbol{\theta}=\sqrt{v_{1}^{2}+v_{2}^{2}+v_{3}^{2}}$.

Moreover, the POE screw theory modelling can be adopted to express the forward kinematics of an open chain robot. For an n-DOF(degrees of freedom) robot, the forward kinematics can be written as shown by Eq.(7).

$$
\boldsymbol{T}=e^{\widehat{\Phi}_{1} \theta_{1}} \cdot e^{\widehat{\Phi}_{2} \theta_{2}} \cdots e^{\widehat{\Phi}_{n} \theta_{n}} \cdot \boldsymbol{T}(0)
$$

Where $\boldsymbol{T}(0)$ represents the initial transformation matrix. The Eq. (12) which represents the POE modelling, can also be used in error modeling of the machine tools.

Error combination by topological structure. The corresponding exponential matrices represent the transformation of these three screws, and $\boldsymbol{e}^{\widehat{\Phi}_{x e}}$ represents the transformation matrix of geometric errors for X-axis as shown in Eq.(7):

$$
\boldsymbol{e}^{\widehat{\boldsymbol{\Phi}}_{x e}}=e^{\widehat{\Phi}_{x x}} \cdot e^{\widehat{\Phi}_{y x}} \cdot e^{\widehat{\Phi}_{2 x}}
$$

The errors modelling for $\mathrm{X}$-axis is:

$$
\boldsymbol{T}^{x}=e^{x \widehat{\Phi}_{x}} \cdot e^{\widehat{\Phi}_{x e}}=e^{x \widehat{\Phi}_{x}} \cdot e^{\widehat{\Phi}_{x x}} \cdot e^{\widehat{\Phi}_{y x}} \cdot e^{\widehat{\Phi}_{2 x}}
$$

Under ideal conditions, errors do not exist. The order of modelling is in the following sequence: $X_{i} \rightarrow Y_{i} \rightarrow Z_{i}$. The ideal transformation matrix, namely the ideal POE model, $\boldsymbol{T}_{\boldsymbol{i}}$ is obtained as,

$$
\boldsymbol{T}_{\boldsymbol{i}}=e^{-x \widehat{\Phi}_{X_{i}}} \cdot e^{-y \widehat{\Phi}_{Y_{i}}} \cdot e^{z \widehat{\Phi}_{Z_{i}}}
$$

With the error twists, squareness errors, linear errors, the actual POE model $T_{a}$ of the machine 
tool can be written as Eq. (10):

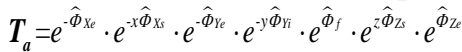

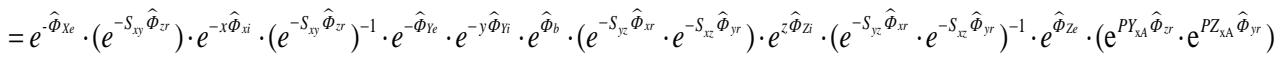

$$
\begin{aligned}
& \widehat{\Phi}_{f}=[0,0,0,0,0,0]^{T} \text { i represents the machine bed. }
\end{aligned}
$$

The tool tip error is the deviation between the ideal and the actual homogeneous coordinates of the tool tip. And the error transformation matrix $\boldsymbol{E}$, can be written as:

$$
\boldsymbol{E}=T_{i}^{-1} \cdot T_{a}
$$

The three parts of $\boldsymbol{E}$ are $E_{\boldsymbol{x}}, E_{\mathrm{y}}$ and $E_{\mathrm{z}}$.

$$
\left[E_{x}, E_{y}, E_{z}, 1\right]^{T}=\boldsymbol{E} \cdot[0,0,0,1]^{T}
$$

The installation errors for the tool and workpiece are too small to be ignored and are not considered in this paper.

\section{Calculation of timeliness machining accuracy}

The illustrated machine tool is machining the same work-piece continuously for a long period of time. Its accuracy retainability and the influence in workpieces accuracy are the most concerns during this work pattern. To study this problem, the errors of machine tool is measured every week, and sampling inspection is carried out to the work-piece every week. As the above section said, the volumetric error distribution can be calculated with time going by.

From the computational results, the accuracy of 3-axis machine tool is decreasing. At the beginning, the volumetric errors are range from " $-22.68 \mu \mathrm{m}$ to $32.77 \mu \mathrm{m}$ " in the first week. And the No.24 week, the volumetric errors are range from " $-83.52 \mu \mathrm{m}$ to $643.89 \mu \mathrm{m}$ ". Under circumstance where the machine tool works continuously for a longtime without maintenance, the machine tool accuracy is losing day by day. As for the work-pieces, the size accuracy also reveals this problem. As Figure 4 shown, total size is changing and accuracy retainability is lower and lower. The signal $\mathrm{k}$ represents the accuracy of work pieces. And if $\mathrm{k}$ is closing to 1, that means the machine `s accuracy retainability is better. That is:

$$
\mathrm{k}=\left|\frac{d_{\text {actual }}}{d_{\text {standard }}}\right|
$$

Where, $d_{\text {standard }}$ is size of requirement. $d_{\text {actual }}$ is size of actual.

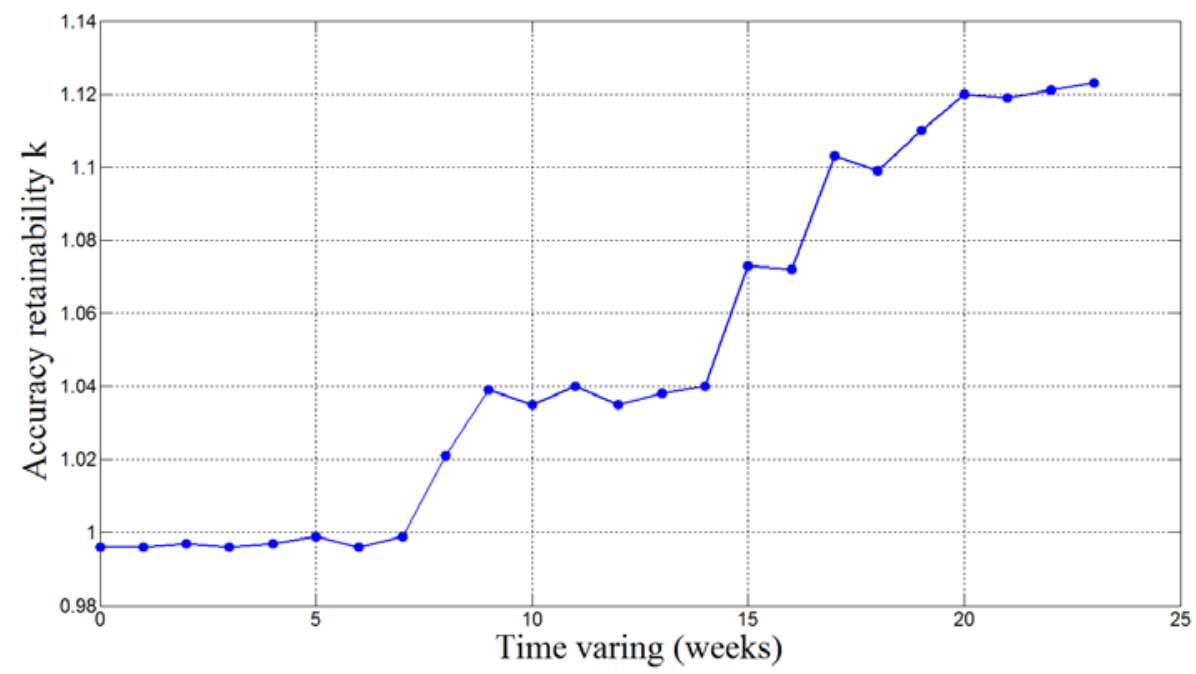

Fig.4 Time-varying accuracy retainability

Figure 4 shows that the accuracy retainability is time-varying. As shown in Figure 4, by measuring the size accuracy of the ladder-workpiece every week, it can be known that the machine tool' machining accuracy is gradually reduced, and the value of the accuracy retainability $\mathrm{k}$ is gradually increased, and it means the machine tool' machining accuracy is reducing. 


\section{Prediction of timeliness accuracy retainability}

In previous sections, the timeliness accuracy retainability is studied. From the results, it can be shown that the accuracy cannot keep unchanged due to continuous and longtime repetitive machining. The machine needs maintenance and adjustment, and only in this way the machine can work as well. Therefore, how to predict the timeliness accuracy retainability of machine tool is a difficult problem faced to manufacturers and users. In this section, a timeliness accuracy retainability prediction method based on LS-SVM is proposed.

Principles of LS-SVM. The loss function is the foundation of the least squares linear system. Its principle is that inequality constraint changes to equality constraint, and there is a set of equations which are deemed as targets. In this paper, LS-SVM method is used as prediction method to predicte timeliness accuracy retainability. Here, time $(t)$ and accuracy retainability $\left(d_{\text {actual }}\right)$ are controlling elements in LS-SVM method. The prediction model is

$$
\left.\begin{array}{lr}
\min & J(\omega, \xi)=\frac{1}{2} \omega^{T} \omega+\gamma\left(\frac{1}{2} \sum_{i=1}^{l} \xi_{i}^{2}\right) \\
\text { s.t. } & d_{i m}=\omega^{T} \phi\left(x_{i}, t, d_{\text {standard }}\right)+b+\xi_{i}
\end{array}\right\}
$$

Where, $x_{i}$ is coordinate position; $t$ is the time; $d_{\text {standard }}$ is the standard size; $d_{i m}$ is improvement coordinate position; $\xi_{i}$ is the residual variable $\xi_{i} \in R ; \phi(\cdot)$ is kernel functions for space mapping: $\phi(\cdot): \mathrm{R}^{n} \rightarrow \mathrm{R}^{n h} ; \omega$ is weight vector, $\omega \in \mathrm{R}^{n h} ; \gamma$ is adjustable parameter; $b$ is deviation value. And termination condition is set as $\left|d_{i m}-d_{\text {standard }}\right| \leq 0.05 \mathrm{~mm}$. And importing Lagrangian function for solving model:

$$
L=\frac{1}{2} \omega^{T} \omega+\gamma\left(\frac{1}{2} \sum_{i=1}^{l} \xi_{i}^{2}\right)-\sum_{i=1}^{l} \alpha_{i}\left(\omega^{T} \phi\left(x_{i}, t, d_{\text {standard }}\right)+b+\xi_{i}-d_{i m i}\right)
$$

Where, $\alpha_{i}$ is Lagrangian.

According existence of extremum, an equation set can be calculated.

$$
\left.\begin{array}{l}
\frac{\partial L}{\partial \omega}=0 \rightarrow \omega=\sum_{i=1}^{l} \alpha_{i} \phi\left(x_{i}, t, d_{\text {standard }}\right) \\
\frac{\partial L}{\partial b}=0 \rightarrow \sum_{i=1}^{l} \alpha_{i}=0 \\
\frac{\partial L}{\partial \xi_{i}}=0 \rightarrow \alpha_{i}=\gamma \xi_{i} \\
\frac{\partial L}{\partial \alpha_{i}}=0 \rightarrow d_{i m}=\omega^{T} \phi\left(x_{i}, t, d_{\text {standard }}\right)+b+\xi_{i}
\end{array}\right\}
$$

The Eq.30 will cut the terms of $\omega$ and $\xi$ down. And then,

$$
\left[\begin{array}{cccc}
0 & 1 & \cdots & 1 \\
1 & K\left(\left(x_{1}, t_{1}, d_{\text {standard }}\right),\left(x_{1}, t_{1}, d_{\text {standard }}\right)\right)+1 / \gamma & \cdots & K\left(\left(x_{1}, t_{1}, d_{\text {standard }}\right),\left(x_{l}, t_{l}, d_{\text {standard }}\right)\right) \\
\vdots & \vdots & & \vdots \\
1 & K\left(\left(x_{l}, t_{l}, d_{\text {standard }}\right),\left(x_{1}, t_{1}, d_{\text {standard }}\right)\right) & \cdots & K\left(\left(x_{l}, t_{l}, d_{\text {standard }}\right),\left(x_{l}, t_{l}, d_{\text {standard }}\right)\right)+1 / \gamma
\end{array}\right] \cdot\left[\begin{array}{c}
b \\
\alpha_{1} \\
\vdots \\
\alpha_{l}
\end{array}\right]=\left[\begin{array}{c}
0 \\
d_{i m 1} \\
\vdots \\
d_{i m l}
\end{array}\right]
$$

According to Mercer condition, the kernel function $K\left(\left(x, t, d_{\text {standard }}\right),\left(x_{l}, t_{l}, d_{\text {standard }}\right)\right)$ for regression estimation of LS-SVM:

$$
f\left(x, t, d_{\text {standard }}\right)=\sum_{i=1}^{l} \alpha_{i} K\left(\left(x, t, d_{\text {standard }}\right),\left(x_{i}, t_{i}, d_{\text {standard }}\right)\right)+b
$$

Where, $\alpha, b$ can be calculated by Eq.31. And the kernel function is arbitrary function of 
symmetric which satisfies Mercer condition. Usually, the radial basis function (RBF) is used. The kernel function

$K\left(\left(x_{i}, t_{i}, d_{\text {standard }}\right),\left(x_{j}, t_{j}, d_{\text {standard }}\right)\right)=\exp \left[-\left(\left(x_{j}, t_{j}, d_{\text {standard }}\right)-\left(x_{i}, t_{i}, d_{\text {standard }}\right)\right)^{2} /\left(2 \sigma^{2}\right)\right]$, there is an undetermined parameter $\sigma$ which is bigger, and whose rate of convergence is faster. Therefore, the $\mathrm{RBF}$ is used which is mainly influenced by regularization parameter $\gamma$ and the width of kernel function $\sigma$. And the two main parameters decide learning ability and generalization ability of LS-SVM.

Simulation of machining accuracy retainability prediction. The LS-SVM for prediction model is shown as following. The RBF is selected for kernel function, and let $\gamma=49.8, \sigma^{2}=0.2$. The prediction model of timeliness accuracy retainability is calculated. The machine tool' actual accuracy retainability is obtained by measuring the size of workpiece every week, and then the thirteenth to twenty-fourth weeks' accuracy retainability data is predicted based on first to twelfth weeks' accuracy retainability data. Figure 5 shows the difference of the measured results and the model prediction results. Figure 6 is the residual error picture of the measured results and the prediction results.

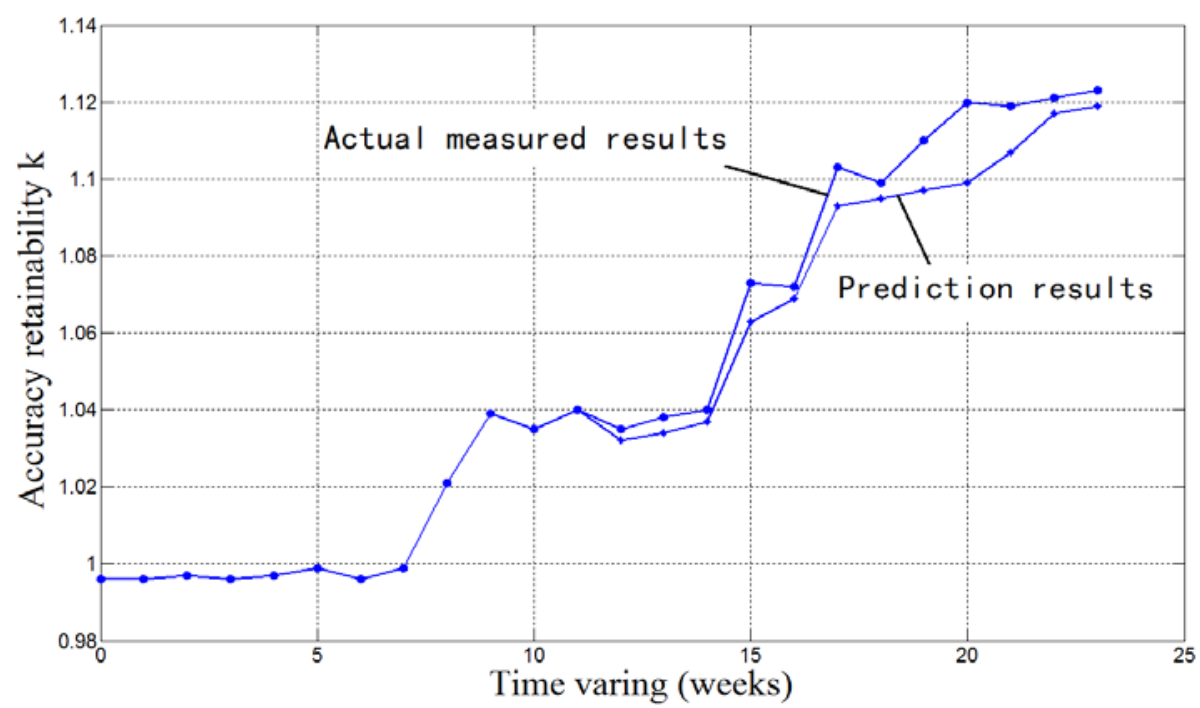

Fig.5 Difference of the measured results and the model prediction results

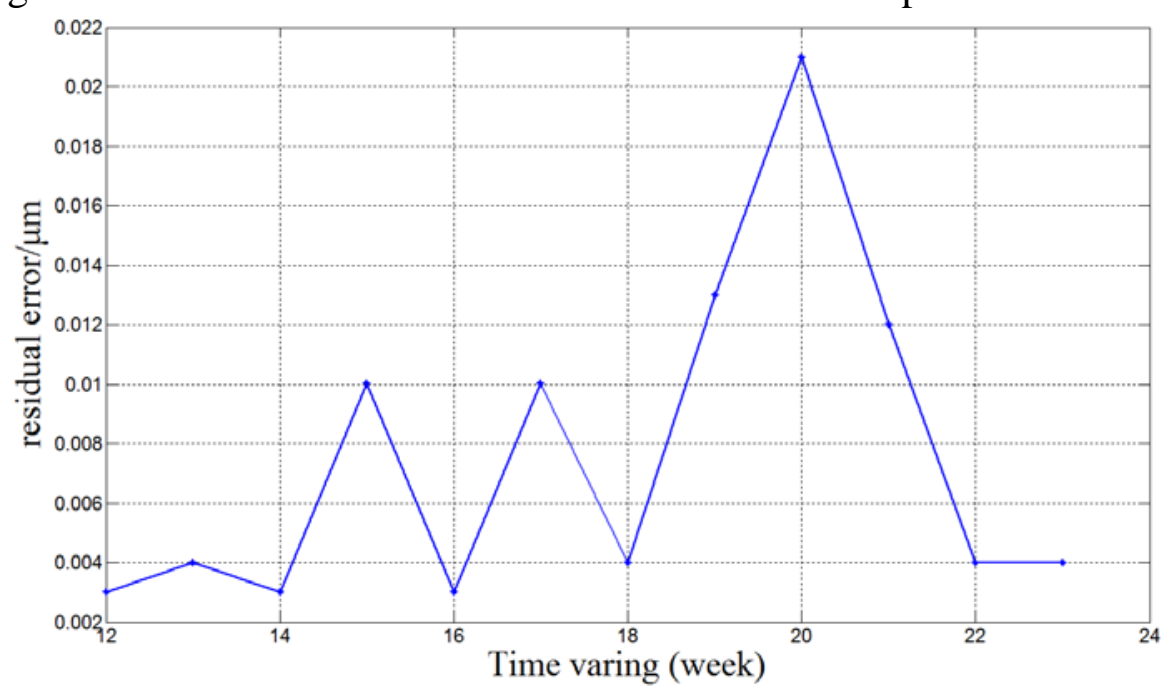

Fig.6 Residual error of the measured results and the prediction results

As is shown in Figure 6, the residual error' maximum value of the measured results and the prediction results is 0.021 , and the difference is very small, so the prediction model proposed in this paper has a relatively higher prediction accuracy. Therefore, the prediction method based on LS-SVM is very effective in predicting the machining accuracy retainability of the machine tool, and it also has important guiding significance for the error compensation of the machine tool. 


\section{Conclusion}

Generally, in order to know about the accuracy retainability of machine tool, it needs to measure the many error sources termly during the specified period. Therefore, in this paper, a solution to improve the machining accuracy retainability based on large amount of error data is proposed. By theoretical derivation and simulation analysis, there are two points to this paper.

1. The POE screw theory is used to establish the comprehensive error model, which can express the changing relationship of machine accuracy with time. The timeliness of accuracy retainability is analyzed, which lays a foundation to establish the prediction model.

2. The prediction model based on LS-SVM method is proposed to predict accuracy retainability with timeliness. And the simulation results show that this proposed method is efficient.

\section{References}

[1] Uddin, M. Sharif, et al. "Prediction and compensation of machining geometric errors of five-axis machining centers with kinematic errors." Accuracy Engineering 33.2 (2009): 194-201.Reference to a book:

[2] Kvrgic, Vladimir, et al. "A control algorithm for improving the accuracy of five-axis machine tools." International Journal of Production Research 52.10 (2014): 2983-2998.

[3] Yan, S., B. Li, and J. Hong, "Bionic design and verification of high-precision machine tool structures." The International Journal of Advanced Manufacturing Technology (2015): 1-13.

[4] Cheng, Q., Luo, R., Gu. P., et al., "Load Induced Error Identification and Camber Curve Design of a Large-Span Crossbeam." Advances in Mechanical Engineering 5 (2013): 847194.

[5] Hsu, Y. Y., and S. S. Wang. "A new compensation method for geometry errors of five-axis machine tools." International journal of machine tools and manufacture 47.2 (2007): 352-360.

[6] Zhu, Shaowei, et al. "Integrated geometric error modeling, identification and compensation of CNC machine tools." International Journal of Machine Tools and Manufacture 52.1 (2012): 24-29.

[7] Chen, I-Ming, et al. "Local POE model for robot kinematic calibration." Mechanism and Machine Theory 36.11 (2001): 1215-1239.

[8] V. Vapnik, "The nature of statistical learning theory." Springer-Verlag, New York, 1995.

[9] $\mathrm{Fu} \mathrm{G}, \mathrm{Fu}$ J, Shen $\mathrm{H}$, et al. Product-of-exponential formulas for precision enhancement of five-axis machine tools via geometric error modeling and compensation. The International Journal of Advanced Manufacturing Technology, (2015): 1-17. 